\title{
COMO CONSTRUIR CONHECIMENTO SOBRE O TEMA DE PESQUISA? APLICAÇÃO DO \\ PROCESSO PROKNOW-C NA BUSCA DE LITERATURA SOBRE AVALIAÇÃO DO DESENVOLVIMENTO SUSTENTÁVEL
}

\author{
Michele Hartmann Feyh Afonso \\ Mestranda em Engenharia de Produção da Universidade Federal de Santa Catarina - UFSC \\ michele_feyh@yahoo.com.br \\ Juliane Vieira de Souza \\ Doutoranda em Engenharia de Produção da Universidade Federal de Santa Catarina - UFSC \\ juliane.vieiradesouza@gmail.com

\section{Sandra Rolim Ensslin} \\ Doutora em Engenharia de Produção pela Universidade Federal de Santa Catarina - UFSC \\ Professora da Universidade Federal de Santa Catarina - UFSC \\ sensslin@gmail.com
}

\section{Leonardo Ensslin}

Doutor em Engenharia Industrial e Sistemas pela University of Southern California - USC Professor da Universidade Federal de Santa Catarina - UFSC leonardoensslin@gmail.com

\section{RESUMO}

Estruturar uma revisão de literatura com o objetivo de construir conhecimento e selecionar periódicos disponibilizados no Portal da CAPES para fundamentação teórica é um desafio para o pesquisador. Diante desse fato, o trabalho proposto apresenta o processo de seleção do portfólio bibliográfico e o processo de análise bibliométrica aplicados no campo de avaliação de desempenho em sustentabilidade, possibilitando a análise de características deste portfólio, tais como periódicos onde o tema é mais recorrente e artigos que se destacam. A pesquisa observa que a publicação de artigos científicos na área é ainda bastante incipiente, mas tem apresentado grande crescimento na última década, e levanta as principais características com relação a autores, periódicos e relevância científica das publicações. Neste contexto, a metodologia ProKnow-C se mostra como uma ferramenta de grande validade para a construção de conhecimento em determinado campo de pesquisa, proporcionando um procedimento estruturado, rigoroso e que minimiza o uso de aleatoriedade e subjetividade no processo de revisão bibliográfica.

Palavras-chave: Avaliação de desempenho; Bibliometria; Desenvolvimento sustentável; Revisão de literatura; Sustentabilidade.

\section{BUILDING KNOWLEDGE ON THEMED RESEARCH: USING THE PROKNOW-C PROCESS TO FIND LITERATURE ON SUSTAINABLE DEVELOPMENT}

\begin{abstract}
When a researcher structures a literature review in order to build knowledge in a particular area, she often selects periodicals from those available on Capes' Portal de Periodicos to support her research with a theoretical basis. This is a challenging task. In light of this, this paper presents the processes of literature selection and bibliometric portfolio analysis that are applied in the field of performance evaluation in sustainability. This analysis enables researchers to identify portfolio characteristics, helping them to separate out the journals in which the themes they're investigating are more often found. The study at hand shows that the publication of scientific papers in this field is still very low, but that is has shown great growth in the last decade. It presents the main characteristics with respect to the authors, journals and scientific relevance of the publications. ProKnow-C is shown to be a tool of high validity for the construction of knowledge in a particular field of research, providing a structured and rigorous procedure, which minimizes the use of randomness and subjectivity in the process of literature review.
\end{abstract}

Keywords: Sustainable development; Sustainability; Performance evaluation; Literature review; Bibliometry. 


\section{INTRODUÇÃO}

O primeiro passo do pesquisador na busca por desenvolver um trabalho e construir conhecimento em determinado contexto, geralmente, se inicia pela revisão da literatura acerca do tema. A revisão da literatura ocupa posição introdutória no desenvolvimento de um projeto de pesquisa e retoma o conhecimento científico acumulado sobre o tema. E, para além da construção do conhecimento acerca do problema de pesquisa, a revisão de literatura permite ao pesquisador avaliar a pertinência científica e não-redundância do trabalho proposto (Macedo, 1994) e define as bases intelectuais em que a lógica da pesquisa está sendo estruturada (Trentini \& Paim, 1999). Para Echer (2001), é somente depois de uma revisão de literatura que o pesquisador terá condições de formar um discurso científico que reflita uma posição a respeito do tema e saberá avaliar a relevância do trabalho proposto, evitando o risco de estar "reinventando a roda" (Macedo, 1994) ou de estar sugerindo uma solução para um problema já resolvido, ou que já apresente melhores soluções na literatura.

No entanto, conduzir uma revisão de literatura, de forma consistente com o tema de pesquisa, está longe de ser considerada uma tarefa trivial. Muito facilmente o pesquisador, especialmente o de primeira viagem, poderá sentir-se perdido na busca e seleção de informações e acabar deixando de lado literatura relevante enquanto atém-se em leituras não-essenciais ao conhecimento desejado.

O Portal de Periódicos da Coordenação de Aperfeiçoamento de Pessoal de Nível Superior (Capes) se constitui hoje na mais completa fonte de artigos científicos disponível no Brasil à disposição dos pesquisadores vinculados à instituições de ensino superior. Trata-se de uma biblioteca virtual que armazena mais de 26.000 títulos e que apresentou mais de 42 milhões de acessos em 2010 (Capes, 2011a) ${ }^{1}$.

A vasta quantidade de conteúdo científico disponível para consulta pode animar o pesquisador mais entusiasmado, mas representa um problema diante da limitação de assimilação da informação; é preciso selecionar apenas parte do conteúdo disponível para construir conhecimento sobre o tema de pesquisa. Essa "superoferta de informação" leva o pesquisador a refletir e pensar maneiras de extrair, dessa grande massa de informação disponível, o conhecimento necessário (Penteado, 2005).

No processo de seleção da literatura de referência é imprescindível que o pesquisador não se deixe levar por caminhos aleatórios, uma vez que esse tipo de pesquisa requer alto grau de vigilância epistemológica, de observação e de cuidado na escolha e no direcionamento dos procedimentos metodológicos. Estes, por sua vez, necessitam de critérios claros e bem definidos, que devem ser constantemente avaliados e redefinidos à medida que se constrói a busca por soluções ao objeto de estudo proposto (Lima e Mioto, 2007).

Este trabalho está estruturado em seis seções, sendo esta primeira seção de caráter introdutório acerca do tema de pesquisa. A segunda, apresenta a fundamentação teórica e os objetivos do trabalho. A terceira, introduz a metodologia empregada no trabalho em termos do enquadramento metodológico da pesquisa e dos procedimentos utilizados. A quarta, apresenta a aplicação detalhada da metodologia, evidenciando todas as etapas para a obtenção do portfólio bibliográfico, e a análise bibliométrica realizada. A quinta, as conclusões obtidas com o trabalho e recomendações para trabalhos futuros, e a sexta, relaciona a bibliografia utilizada.

\footnotetext{
${ }^{1} \mathrm{O}$ acesso ao Portal de Periódicos da Capes é disponibilizado apenas a uma parte das instituições de ensino superior brasileiras, incluindo todas as instituições federais. O acesso é disponibilizado gratuitamente apenas para os pesquisadores vinculados a essas instituições (Capes, 2011b).
}

Revista de Gestão Social e Ambiental - RGSA, São Paulo, v. 5, n. 2, p. 47-62, mai./ago. 2011. 


\section{FUNDAMENTAÇÃO TEÓRICA}

A problemática apresentada abrange diversas áreas do conhecimento, entre as quais está aquela que embasa o objetivo deste trabalho: avaliação de desempenho no contexto do desenvolvimento sustentável. O relatório Brundtland, elaborado pela World Commission on Environment and Development [WCED], define o desenvolvimento sustentável como "[...]aquele capaz de permitir a satisfação das necessidades da geração presente sem comprometer a satisfação das gerações futuras" (1987, p.8). Segundo Andrew W. Savitz, presidente da Sustainable Business Strategies, sustentabilidade é "[...]o ponto de intersecção entre os negócios e os interesses da sociedade e do planeta" (Conselho Empresarial Brasileiro para o Desenvolvimento Sustentável [CEBDS], 2009, p.16).

No contexto corporativo, este tema é, geralmente, abordado sob um enfoque mais específico, considerando o conceito de Triple Bottom Line definido por Elkington (1999). De acordo com esta definição, a aplicação dos valores de sustentabilidade nas empresas considera três dimensões que devem estar em equilíbrio: econômica, social e ambiental. Embora esta questão tenha atingido grande notoriedade no mundo corporativo, exigindo mudanças reais nos formatos de produção e de trabalho, está longe de se restringir a este campo. O governo, pela definição de políticas públicas, e os indivíduos, por meio de suas opções de consumo, se constituem em atores essenciais na busca pelo desenvolvimento sustentável.

A complexidade conceitual do tema e sua característica dinâmica de adaptação a cada contexto tornam este um dos conceitos mais difíceis de serem mensurados quanto ao seu desempenho. Tal dificuldade é abordada e discutida por Delai e Takahashi (2008) e por Vasconcelos, Andrade e Cândido (2009) em diferentes campos de pesquisa. Nestes termos, emerge a seguinte pergunta de pesquisa: como realizar a seleção da literatura de referência que gere o conhecimento demandado pelo pesquisador no início de uma pesquisa sobre avaliação de desempenho em desenvolvimento sustentável a fim de proporcionar condições necessárias para, em um segundo momento, buscar as oportunidades de contribuição ao tema?

A busca por respostas para esta questão se constitui no foco deste trabalho, que tem por objetivo construir o conhecimento necessário para orientar o pesquisador na busca de oportunidades (lacunas) de pesquisa no contexto de avaliação de desempenho em desenvolvimento sustentável. Assim, os objetivos específicos da pesquisa se constituem em: 1) selecionar as palavras-chave adequadas para a pesquisa; 2) selecionar as bases de dados relativas ao tema de pesquisa; 3 ) proceder a busca de artigos alinhados com o tema de pesquisa; 4) identificar os artigos relevantes da amostra selecionada; e 5) analisar os resultados obtidos com relação a relevância científica e saliência de autores e periódicos de destaque.

A construção do conhecimento sobre o tema de pesquisa será embasada na metodologia ProKnow-C (Knowledge Development Process - Constructivist) (Ensslin, L., Ensslin, S. R., Lacerda, \& Tasca, 2010d) desenvolvida pelo Laboratório de Metodologias Multicritério em Apoio à Decisão (LabMCDA) ${ }^{2}$, que apresenta aplicações recentes em outros campos de pesquisa, tais como Gestão Púbica (Valmorbida, Ensslin, L., \& Ensslin, S. R., 2011), Evidenciação Ambiental (Rosa, Ensslin, S. R., \& Ensslin, L., 2009), Avaliação de Desempenho Organizacional (Bortoluzzi \& Ensslin, 2010) e Avaliação de Programas de Treinamento (Tasca, Ensslin, L., Ensslin, S. R., \& Alves, 2010).

O processo ProKnow-C se constitui em uma metodologia de construção do conhecimento estruturada em quatro etapas: 1) seleção do portfólio bibliográfico que proporcionará a revisão de literatura; 2) análise bibliométrica do portfólio bibliográfico; 3) análise sistêmica do portfólio

\footnotetext{
${ }^{2}$ O Laboratório de Metodologias Multicritério em Apoio a Decisão consiste em um grupo de estudos de pesquisadores vinculados ao Programa de Pós-Graduação em Engenharia da Produção da Universidade Federal da Santa Catarina (Brasil). Para mais informações, consulte: http://www.eps.ufsc.br/labmcda/.
}

Revista de Gestão Social e Ambiental - RGSA, São Paulo, v. 5, n. 2, p. 47-62, mai./ago. 2011. 
bibliográfico; 4) elaboração dos objetivos de pesquisa. Neste trabalho, serão aplicadas as etapas 1 e 2 do processo ${ }^{3}$.

A metodologia adotada proporcionará, ao fim do trabalho, obter um portfólio bibliográfico relevante ao tema de pesquisa, que será selecionado sob critérios definidos objetivamente, buscando manter os artigos de maior relevância científica ao tema neste portfólio (Ensslin, L., Ensslin, S. R., Lacerda, \& Tasca, 2010c). Com base no portfólio de artigos científicos obtido, será realizada uma análise bibliométrica, que consiste no levantamento de estatísticas de um conjunto definido de artigos para a gestão da informação e do conhecimento científico do tema de pesquisa (Ensslin, L., Ensslin, S. R., Lacerda, \& Tasca, 2010a). Tal procedimento é definido como fase posterior à seleção do portfólio bibliográfico na metodologia definida por Ensslin, L. et al. (2010a) e segue uma sequência de passos estruturados em sua elaboração.

\section{MÉTODO DE PESQUISA}

A metodologia utilizada evidencia, em um primeiro momento, o enquadramento metodológico da pesquisa, apresentando as características, propriedades e limitações do trabalho. Em um segundo momento, é apresentada a metodologia adotada para atingir os objetivos propostos.

\subsection{Enquadramento metodológico}

O enquadramento metodológico da pesquisa deste trabalho será apresentado sob o enfoque de sete dimensões: natureza do objetivo; natureza da pesquisa; coleta de dados; abordagem do problema; resultados; população e amostra; e instrumento de intervenção.

Quanto à natureza do objetivo, a metodologia adotada se caracteriza como exploratória, pois se constitui em um primeiro levantamento sobre o tema, sem finalidade de confirmar alguma hipótese; e descritiva, pois apresenta, de forma descritiva, os procedimentos seguidos e resultados obtidos.

Com relação à natureza da pesquisa, este trabalho é classificado como levantamento teórico/ilustrativo, pois busca a reflexão sobre o problema, além de apresentar uma aplicação prática com base em um processo estruturado.

No que concerne à coleta de dados, este trabalho é realizado com base em pesquisa de dados secundários, que se constituem em artigos científicos disponibilizados gratuitamente nas bases de dados indexadas ao Portal da Capes.

Quanto à abordagem do problema, a pesquisa é definida como qualitativa, devido à estrutura de coleta de dados secundários e sua análise quanto à permanência no portfólio bibliográfico, e também quantitativa, no que concerne à análise bibliométrica do conjunto de artigos obtidos.

Com relação aos resultados obtidos, a pesquisa se classifica como aplicada, pois são coletados dados com base em um procedimento estruturado, que, posteriormente, são analisados seguindo também uma metodologia estruturada.

No que diz respeito à população e amostra, são considerados como população-alvo do trabalho toda publicação científica em periódico que abarque o tema de pesquisa e que esteja disponível para acesso gratuito no Portal da Capes. A amostra utilizada neste trabalho teve como base artigos selecionados em quatro bases de dados que possuem maior relação com o tema de pesquisa e maior quantidade de publicações na área, a partir dos quais o procedimento de seleção do portfólio bibliográfico foi aplicado.

$\mathrm{O}$ instrumento de intervenção utilizado neste trabalho é parte da metodologia ProKnow-C proposta pelo LabMCDA, que estabelece os procedimentos de seleção e análise dos resultados adotados.

\footnotetext{
${ }^{3}$ Para obter mais informações sobre as etapas 3 e 4, consulte Ensslin, L., Ensslin, S. R., Lacerda, \& Tasca (2010b) e Lacerda, Ensslin, L., \& Ensslin S. R., (2011a).
}

Revista de Gestão Social e Ambiental - RGSA, São Paulo, v. 5, n. 2, p. 47-62, mai./ago. 2011. 


\subsection{Procedimento de seleção do portfólio bibliográfico}

A metodologia de seleção do referencial bibliográfico ProKnow-C, definida pelo LabMCDA, consiste em uma série de procedimentos sequenciais que se iniciam desde a definição do mecanismo de busca de artigos científicos a ser utilizado, seguindo por uma série de procedimentos pré-estabelecidos até atingir a fase de filtragem e seleção do portfólio bibliográfico relevante sobre o tema. O procedimento é apresentado de forma resumida na Figura 1, e uma descrição de cada etapa será apresentada a seguir.

Definidos os eixos de pesquisa, isto é, o tema que determinará a pesquisa que será realizada, se faz necessário estipular quais serão as palavras-chave utilizadas nos mecanismos de busca a fim de se obter os artigos científicos no tema desejado. Essa pode não ser uma tarefa simples, uma vez que estabelece quais artigos serão selecionados para entrada no processo e quais serão excluídos deste processo. Nas fases posteriores do processo, o pesquisador poderá verificar se as palavras selecionadas estão, de fato, conseguindo discriminar os artigos científicos referentes à área de pesquisa, e não encontrando sucesso, deve-se retroceder no processo até este ponto e selecionar novas palavras-chave.

As palavras-chave selecionadas devem ser utilizadas nos mecanismos de busca de artigos científicos, isoladamente ou combinadas. Como não há um mecanismo que faça a busca nas diversas bases de dados existentes - onde o conteúdo dos periódicos fica armazenado - é preciso selecionar quais as bases de dados serão utilizadas para fazer o procedimento de busca dos artigos. Esta definição é feita pelo pesquisador, que poderá melhor avaliar quais são as bases de dados que possuem maior consistência com o tema de pesquisa. No caso de o pesquisador não conseguir definir quais são as melhores bases de dados a considerar para o tema de pesquisa, pode-se proceder uma busca com as palavras-chave em diversas bases de dados, para então verificar quais delas apresentam maior representatividade do tema de pesquisa, isto é, quais possuem uma maior quantidade de artigos científicos disponíveis na área de interesse, para, então, optar pelas bases de dados a serem utilizadas na pesquisa.

A busca das palavras-chave estipuladas nas bases selecionadas pode ser realizada individualmente ou conjuntamente, se o tema contemplar mais de um eixo de pesquisa. Os resultados obtidos em cada base de dados podem ser exportados para um software de gerenciamento bibliográfico para facilitar a manipulação das informações e padronizar seu formato de apresentação ${ }^{4}$.

Com o objetivo de verificar se as palavras-chave selecionadas estão adequadas ao tema de pesquisa, sugere-se a leitura de dois artigos entre aqueles obtidos na pesquisa. Esta leitura permitirá identificar se as palavras-chave estão adequadas ou se há outros termos que de representem melhor o tema de pesquisa, neste caso, será necessário incorporá-los na busca pelos artigos científicos.

\footnotetext{
${ }^{4} \mathrm{O}$ pesquisador perceberá que não há um padrão de apresentação da informação entre as diferentes bases de dados, nem mesmo é observado um padrão para o procedimento de pesquisa. Diante desta situação, os softwares de gerenciamento bibliográfico se mostram como ferramentas de grande auxílio no processo de manipulação da informação coletada em diferentes bases de dados.
} 


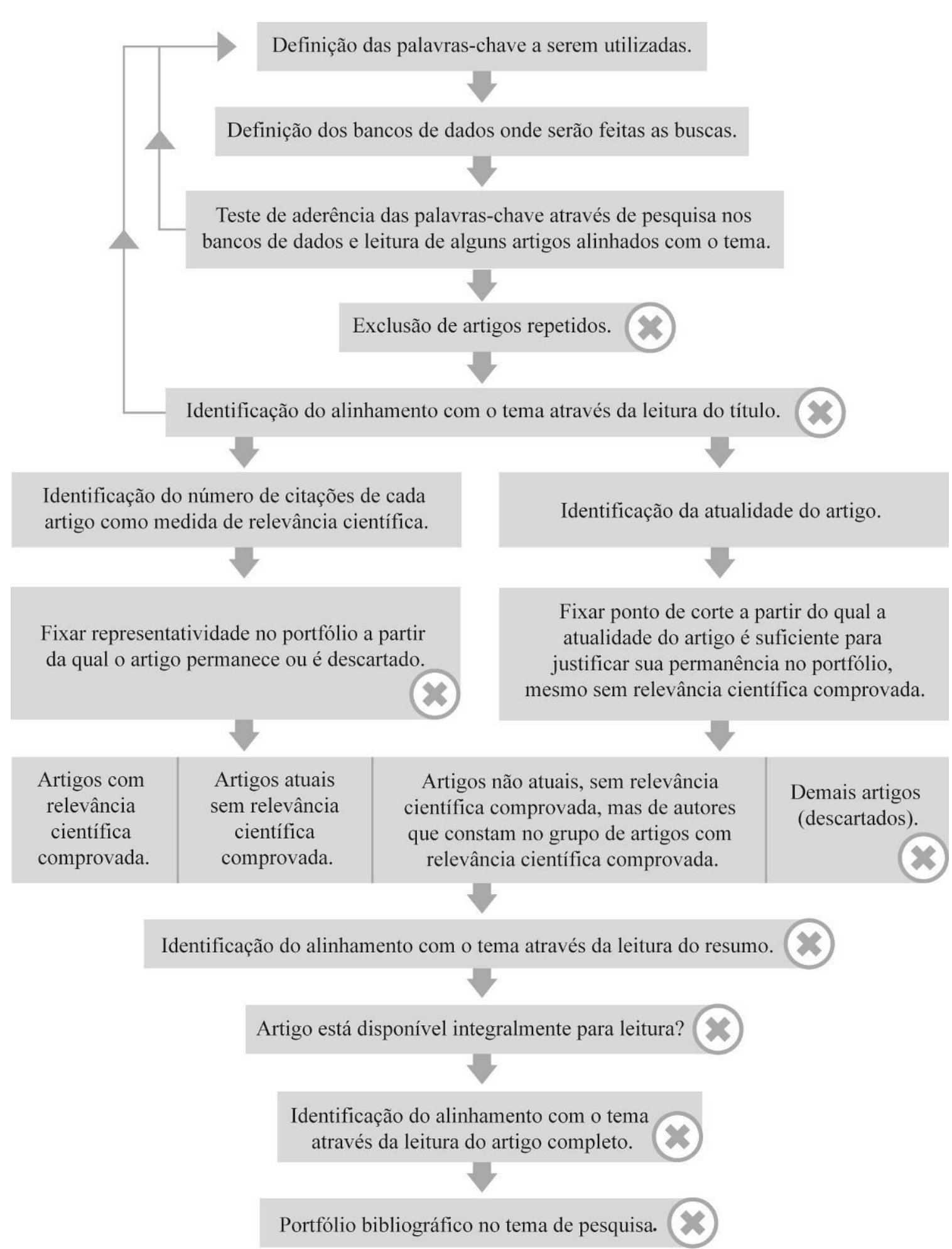

Legenda

$\begin{aligned} & \text { Não obtendo bons } \\ & \text { resultados, retornar } \\ & \text { ao ponto inicial. }\end{aligned}$
(3) escartados artigos
do portfólio atual.

Figura 1: Resumo do processo de seleção do portfólio bibliográfico da metodologia de construção do conhecimento ProKnow-C.

Fonte: Ensslin, L. et al. (2010c, 2010d). 
Após a verificação da adequabilidade das palavras-chave ao tema de pesquisa e realizada a busca de artigos nas bases de dados selecionadas, têm-se então o banco de artigos bruto, que possibilitará que seja feito o processo de filtragem para se chegar a um portfólio bibliográfico relevante e mais reduzido.

A primeira etapa na fase de filtragem do banco de artigos bruto se refere à exclusão dos artigos repetidos. Como a pesquisa por artigos, é realizada em diversas bases, é comum que o conjunto de artigos reunido pelo pesquisador contenha artigos repetidos. A segunda etapa da filtragem consiste na leitura dos títulos dos artigos, a partir da qual deve ser descartada parte dos artigos que atualmente estão no banco de artigos formado. Esta etapa se justifica pelo fato de que frequentemente a busca das palavras-chave é realizada não somente nos títulos e palavras-chave dos artigos, mas também nos resumos, possibilitando que sejam incorporados ao banco de artigos do pesquisador trabalhos que, apesar apresentarem presença das palavras-chave procuradas, não tratem especificamente do tema de pesquisa.

A terceira etapa da filtragem consiste na verificação da relevância científica dos artigos selecionados como critério manutenção destes no banco de artigos do pesquisador. Tal avaliação não representa uma tarefa simples, envolvendo diversos passos e uma análise crítica do pesquisador sobre os artigos reunidos até o momento. O primeiro passo na busca pela relevância científica se constitui na busca da quantidade de citações que cada artigo possui. Esta busca pode ser realizada através da ferramenta de busca Google Acadêmico, a qual apresenta o número de citações de cada artigo facilmente na busca simples pelo título do artigo ${ }^{5}$. De posse desta informação, cabe ao pesquisador estabelecer um ponto de corte a partir do qual os artigos serão classificados como: artigos com reconhecimento científico confirmado ou artigos com reconhecimento científico ainda não confirmado. A metodologia recomenda que o pesquisador calcule a representatividade de cada artigo perante o banco de artigos completo em termos do número de citações, e sugere-se que o ponto de corte adotado seja em torno de $85 \%$. No entanto, vale ressaltar que cada tema de pesquisa tem suas características, podendo oscilar tanto na quantidade de artigos disponíveis quanto na quantidade de citações destes artigos, sendo necessário, assim, avaliar cada situação individualmente.

Do grupo de artigos classificado como de reconhecimento científico confirmado passa-se para a etapa de leitura do resumo, que possibilitará que verifique se o artigo está realmente alinhado ao tema de pesquisa e se definirá se o artigo permanece no banco de artigos do pesquisador, ou se será descartado. Estabelecido o grupo de artigos que permanece nesta fase, é realizada uma lista dos autores destes artigos, chamada banco de autores.

Para o grupo de artigos em que a relevância científica ainda não pôde ser confirmada, o primeiro fator a ser considerado neste momento é o ano em que o artigo foi escrito. Se o artigo foi publicado em, no máximo, dois anos, então se passa para a leitura do resumo. Já se o artigo é mais antigo, então, primeiramente, avalia-se se algum dos autores faz parte do banco de autores formado entre os artigos com relevância científica já confirmada; em caso afirmativo, passa-se para a leitura do resumo; em caso negativo, o artigo é descartado. A partir da leitura do resumo, define-se se o artigo está alinhado com o tema de pesquisa e, por consequência, sua permanência ou descarte.

A última etapa da formação do referencial bibliográfico consiste na união dos artigos, cuja relevância científica foi verificada, com os artigos atuais e ainda com aqueles cujos autores fazem parte do banco de autores formado, e a consequente pesquisa pela disponibilidade dos artigos integrais para consulta (até este momento trabalhou-se apenas com os resumos). Se o artigo não estiver disponível integralmente, é descartado da relação do pesquisador. Para aqueles artigos que

\footnotetext{
${ }^{5}$ A tarefa de buscar a quantidade de citações de cada artigo no Google Acadêmico pode ser bastante trabalhosa, dependendo da quantidade de artigos que se tenha neste ponto do processo. Se o pesquisador tiver maior conhecimento sobre programação, é conveniente considerar a elaboração de uma programação que realize a busca automaticamente.
}

Revista de Gestão Social e Ambiental - RGSA, São Paulo, v. 5, n. 2, p. 47-62, mai./ago. 2011. 
estão disponíveis integralmente, procede-se a leitura integral e define-se, por fim, o alinhamento com o tema de pesquisa. Aqueles artigos considerados alinhados permanecem na relação do pesquisador e passam a compor o portfólio bibliográfico do pesquisador no tema de pesquisa.

\section{ANÁLISE E DISCUSSÃO DOS RESULTADOS}

A aplicação em nosso contexto de pesquisa segue todos os passos descritos na seção anterior e representados na Figura 1. Nos próximos parágrafos, serão descritos, em detalhes, os procedimentos seguidos e as decisões tomadas na aplicação da metodologia para atingir aos objetivos do trabalho.

\subsection{Seleção das palavras-chave}

O objetivo de nosso trabalho é fazer uma revisão de literatura sobre avaliação de desempenho no contexto da sustentabilidade. Entendemos que se tratam de dois eixos de pesquisa, assim definimos palavras-chave para ambos os eixos. Para o eixo relativo à avaliação de desempenho, em um primeiro momento, quatro palavras-chave foram escolhidas: "performance measurement", "performance evaluation", "performance assessment" e "performance appraisal". Para a definição destas palavras-chave utilizou-se como referência a literatura da área (Lacerda, Ensslin, L., \& Ensslin, S. R., 2011b).

A seleção das palavras-chave relativas ao eixo de sustentabilidade passou por um processo mais detalhado. Em um primeiro momento, optou-se por palavras mais específicas, tais como "sustainable development" e "sustainability process" entre outras, que geram uma quantidade muito reduzida de artigos em uma primeira busca realizada em uma série de bases de artigos, totalizando menos de 1.000 artigos encontrados. As buscas por artigos foram realizadas fazendo combinações entre as palavras-chave do primeiro e do segundo eixos de pesquisa. Como esta quantidade foi considerada bastante reduzida, optou-se por uma segunda tentativa em que se buscou utilizar três palavras mais abrangentes: "sustainability", "sustainable" e "sustain", a partir das quais foi possível obter resultados próximos a 2.000 artigos.

Ainda assim, a quantidade de artigos encontrada foi considerada bastante reduzida, fato que nos levou a reavaliar as palavras-chave do eixo de avaliação de desempenho. Após a leitura de alguns artigos da área, observou-se que, se tratando de sustentabilidade, o desempenho é, muitas vezes, medido por meio de indicadores. Assim, foi adicionada a palavra-chave "indicator" na busca, totalizando cinco palavras-chave no eixo de avaliação de desempenho, três palavras-chave no eixo de sustentabilidade e 15 combinações de palavras-chave entre ambos os eixos. Por fim, foram encontrados 9.123 artigos na combinação destas palavras-chave nas bases selecionadas para a pesquisa.

\subsection{Seleção das bases de dados de pesquisa}

As bases de dados a serem utilizadas para a pesquisa dos artigos foram definidas, em uma primeira fase, pela adequação do conteúdo da base ao tema de pesquisa e, em uma segunda fase, pela quantidade de artigos disponíveis no tema de pesquisa. Assim, optou-se por considerar para a composição do portfólio bibliográfico as bases de dados Scopus, ISI Web of Knowledge, Sciende Direct e Ebsco.

Para cada uma destas quatro bases de dados foram realizadas 15 pesquisas, resultantes das combinações entre as palavras-chave de ambos os eixos de pesquisa. A pesquisa foi realizada buscando somente artigos publicados em periódicos nos últimos 10 anos (desde 2001) ${ }^{6}$ que

\footnotetext{
${ }^{6}$ A delimitação de tempo para a pesquisa foi definida pelos autores com o objetivo de buscar a literatura recente na área. Uma breve pesquisa por artigos publicados em periódicos em anos anteriores mostrou que a quantidade de publicações sobre o tema era bastante restrita.
} 
contivessem as combinações de palavras-chave pesquisadas no título, nas palavras-chave ou no resumo do artigo.

Tabela 1: Quantidade de artigos científicos encontrados nas bases de dados de pesquisa.

\begin{tabular}{|l|c|}
\hline Base de Dados & Artigos \\
\hline SCOPUS & 4.117 \\
\hline ISI Web of Knowledge & 2.402 \\
\hline Science Direct & 1.362 \\
\hline EBSCO & 1.242 \\
\hline Total & 9.123 \\
\hline
\end{tabular}

Fonte: Pesquisa realizada nas bases de dados descritas em 19/03/2011.

\subsection{Processo de filtragem}

O primeiro passo do processo de filtragem consiste na identificação dos artigos repetidos, etapa na qual o banco de artigos atual reduziu sua quantidade de 9.123 para 5.119 artigos. No passo seguinte, que se refere à leitura dos títulos dos artigos com o objetivo de verificar a adequabilidade do artigo ao tema de pesquisa, uma grande parte dos artigos é descartada, e permanecem no processo 893 artigos, que foram considerados alinhados com o tema de pesquisa. Nesta etapa, foram considerados os artigos que tratam do tema de sustentabilidade de forma abrangente, considerando, pelo menos, as dimensões social, ambiental e econômica. Assim, foram descartados os artigos que consideram somente uma ou duas dessas dimensões.

O procedimento de pesquisa da relevância científica dos artigos foi realizado por meio da busca de citações de cada artigo no Google Acadêmico. Com base nestas informações, foi calculada a representatividade de cada artigo no conjunto de artigos atual. Estabeleceu-se, como ponto de corte de permanência dos artigos no portfólio atual, uma representatividade de, no mínimo, 60\%, resultando em uma quantidade de 58 artigos classificados como de relevância científica confirmada, que representam $6,5 \%$ do banco de artigos atual. $O$ ponto de corte foi definido abaixo do recomendado pela metodologia $(85 \%)$ devido à restrita quantidade de artigos que seria alocada nesta categoria se esta faixa fosse adotada.

Os 58 artigos classificados como de reconhecimento científico confirmado tiveram seus resumos lidos, a fim de verificar o alinhamento com o tema de pesquisa. Nesta fase, 32 artigos foram descartados e permaneceram no processo 26 artigos. Com base nesta relação de artigos, foi construído um banco de autores que será utilizado no processo de identificação da relevância científica do grupo de artigos que ainda não possui relevância confirmada.

O grupo de artigos com relevância científica ainda não confirmada (835) é avaliado, primeiramente, com relação ao tempo de publicação. Artigos com menos de dois anos de publicação são direcionados para a leitura do resumo (292), enquanto os artigos mais antigos (543) somente são direcionados para a leitura do resumo se tiverem entre seus autores um daqueles que compõem o banco de autores formado com base na relação de artigos com relevância científica confirmada (31); caso contrário, são descartados (512). Assim, 323 artigos tiveram seus resumos lidos com o objetivo de verificar o alinhamento com o tema de pesquisa, dos quais 321 foram descartados e dois artigos permaneceram no processo.

Unindo os artigos que permanecem no processo em ambos os grupos, obtêm-se 28 artigos no portfólio bibliográfico atual. Neste momento, verifica-se a disponibilidade do texto integral deste grupo de artigos, onde se identificou seis artigos cujos textos não estavam disponíveis, que foram 
descartados $^{7}$. Os 22 artigos que permanecem no processo passam para a fase de leitura do texto integral, onde outros nove artigos são descartados por não apresentarem alinhamento com o tema de pesquisa. Por fim, chega-se a um portfólio bibliográfico de 13 artigos relativos ao tema de pesquisa, que são apresentados na Tabela 2.

Tabela 2: Portfólio bibliográfico

\begin{tabular}{|l|l|c|c|}
\hline \multicolumn{1}{|c|}{ Autores } & \multicolumn{1}{|c|}{ Título } & Ano & Citações \\
\hline G. Miller & $\begin{array}{l}\text { The development of indicators for sustainable } \\
\text { tourism: Results of a Delphi survey of tourism } \\
\text { researchers }\end{array}$ & 2001 & 158 \\
\hline F. Figge \& T. Hahn & $\begin{array}{l}\text { Sustainable Value Added - Measuring } \\
\text { corporate contributions to sustainability } \\
\text { beyond eco-efficiency }\end{array}$ & 2004 & 132 \\
\hline $\begin{array}{l}\text { V. Veleva \& M. } \\
\text { Ellenbecker }\end{array}$ & $\begin{array}{l}\text { Indicators of sustainable production: } \\
\text { Framework and methodology }\end{array}$ & 2001 & 88 \\
\hline $\begin{array}{l}\text { C. Labuschagne, A. C. } \\
\text { Brent \& R. P. G. van Erck }\end{array}$ & $\begin{array}{l}\text { Assessing the sustainability performances of } \\
\text { industries }\end{array}$ & 2005 & 85 \\
\hline H. Bossel & $\begin{array}{l}\text { Assessing viability and sustainability: A } \\
\text { systems-based approach for deriving } \\
\text { comprehensive indicator sets }\end{array}$ & 2001 & 76 \\
\hline $\begin{array}{l}\text { S. López-Ridaura, O. } \\
\text { Masera \& M. Astier }\end{array}$ & $\begin{array}{l}\text { Evaluating the sustainability of complex socio- } \\
\text { environmental systems. the MESMIS } \\
\text { framework }\end{array}$ & 2002 & 71 \\
\hline $\begin{array}{l}\text { G. A. Mendoza \& R. } \\
\text { Prabhu }\end{array}$ & $\begin{array}{l}\text { Qualitative multi-criteria approaches to } \\
\text { assessing indicators of sustainable forest } \\
\text { resource management }\end{array}$ & 2003 & 66 \\
\hline D. Krajnc \& P. Glavič & $\begin{array}{l}\text { A model for integrated assessment of } \\
\text { sustainable development }\end{array}$ & 2005 & 60 \\
\hline $\begin{array}{l}\text { E. Ronchi, A. Federico \& F. } \\
\text { Musmeci }\end{array}$ & $\begin{array}{l}\text { A system oriented integrated indicator for } \\
\text { sustainable development in Italy }\end{array}$ & 2002 & 58 \\
\hline A. Boggia and C. Cortina & $\begin{array}{l}\text { Measuring sustainable development using a } \\
\text { multi-criteria model: A case study }\end{array}$ & 2010 & 43 \\
\hline $\begin{array}{l}\text { H. R. Sahely, C. A. } \\
\text { Kennedy and B. J. Adams }\end{array}$ & $\begin{array}{l}\text { Developing sustainability criteria for urban } \\
\text { infrastructure systems }\end{array}$ & $\begin{array}{l}\text { Measurement and evaluation of sustainable } \\
\text { development. A composite indicator for the } \\
\text { islands of the North Aegean region, Greece }\end{array}$ & 2005 \\
\hline $\begin{array}{l}\text { J. Kondyli } \\
\text { Achillas, C. Vlachokostas, } \\
\text { D. Spyridi and K. Nikolaou }\end{array}$ & $\begin{array}{l}\text { information management for the evaluation of } \\
\text { sustainability in urban areas: A system of } \\
\text { indicators for Thessaloniki, Greece }\end{array}$ & 2010 \\
\hline
\end{tabular}

Fonte: Resultado da metodologia aplicada neste trabalho.

\footnotetext{
${ }^{7}$ Nesta fase, foram descartados tanto artigos que não tinham seu texto completo disponível, quanto artigos que, apesar de estarem disponíveis, não estavam disponibilizados gratuitamente no Portal de Periódicos da Capes.
} 


\subsection{Análise bibliométrica do portfólio bibliográfico obtido}

Como parte subsequente da construção do portfólio bibliográfico para o tema de pesquisa, está a análise bibliométrica dos artigos encontrados. Tal análise, tem por objetivo evidenciar informações sobre o portfólio bibliográfico obtido por meio da análise e quantificação de suas características (Ensslin, L. et al., 2010a).

Primeiramente, avaliam-se os periódicos nos quais os artigos estão publicados. Evidencia-se que não há periódicos que concentrem grande parte do portfólio bibliográfico, pelo contrário, os artigos encontram-se diluídos em 11 periódicos diferentes, com destaque para o Ecological Indicators e o Jornal of Cleaner Production, que concentram 2 periódicos cada.

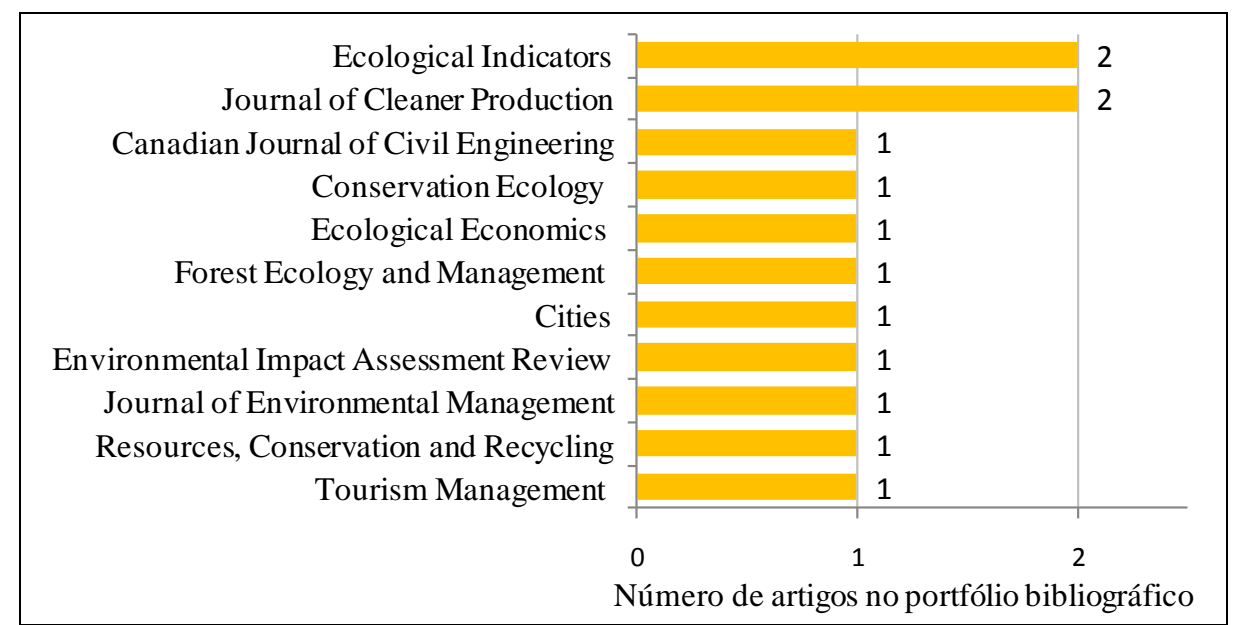

Figura 2: Relevância dos periódicos do portfólio bibliográfico

Fonte: Resultados da pesquisa.

Ao avaliar as 145 referências de artigos publicados em periódicos que estavam contidas nas referencias bibliográficas do portfólio de pesquisa, observa-se também grande variabilidade, uma vez que estão distribuídos em 85 periódicos diferentes. Entre estes periódicos, destaca-se o Ecological Economics, que concentra a maior quantidade de artigos das referências: 15 artigos. Os periódicos que atingiram destaque no portfólio de pesquisa também encontram saliência nas referências, mesmo que de forma secundária, entre eles o Ecological Indicators, que aparece na segunda posição com cinco artigos, e o Jornal of Cleaner Production, que surge na quarta posição com quatro artigos.

Evidencia-se que não há autores de destaque no portfólio de pesquisa, pois todos os autores aparecem uma única vez (veja Tabela 2). Por outro lado, a análise dos autores presentes nas referências dos artigos do portfólio bibliográfico aponta para uma maior concentração de publicações de oito autores: D. Butler, D. Loucks, H. Daly, P.Pearson, S.-L. Huang, T. Foxon, R. Prabdu e V. Veleva, sendo estes dois últimos autores de artigos que constam também no portfólio de pesquisa.

Na busca e análise da relevância científica dos artigos do portfólio bibliográfico, observamse alguns destaques: os artigos de G. Miller e F. Figge et al. apresentam uma quantidade de citações acima da faixa padrão, observada para o total de artigos do portfólio (40 a 90 citações, onde se encontram a maior parte dos artigos). Contrastivamente, encontram-se também dois destaques no outro extremo do gráfico, são os artigos de J. Kondyli e de N. Moussiopoulos et al., que apresentam citações abaixo do padrão do portfólio. Estes artigos se tornaram parte do portfólio, em decorrência do ano de publicação (ambos são de 2010) em conjunto com o alinhamento verificado com tema de pesquisa. 


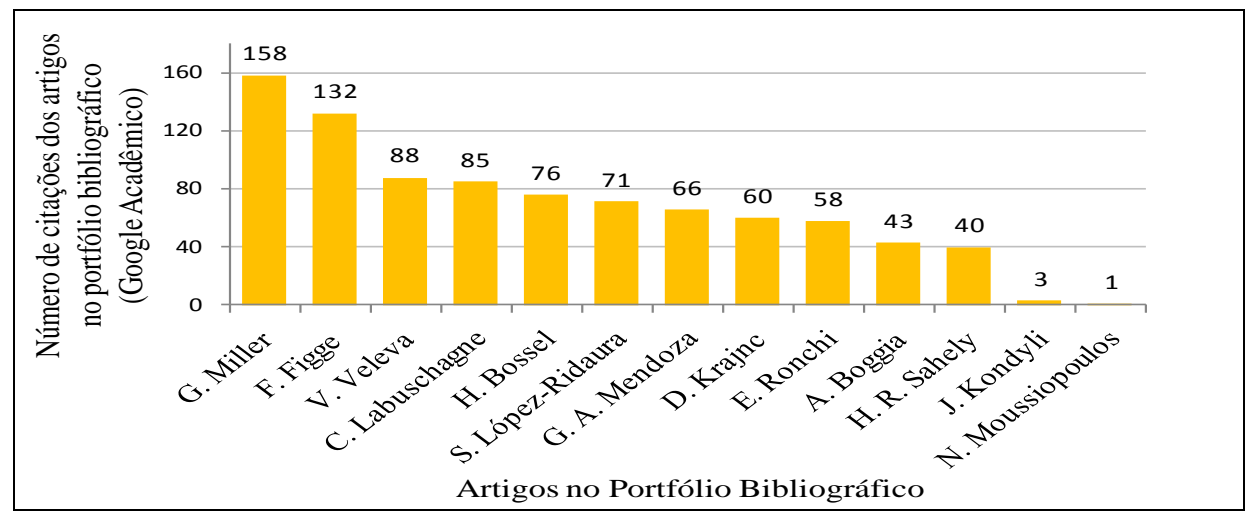

Figura 3: Relevância dos artigos do portfólio bibliográfico.

Fonte: Resultados da pesquisa

Os artigos presentes nas referências dos artigos do portfólio bibliográfico apresentam maior variabilidade na quantidade de citações, obtendo-se artigos com citações que vão de zero a 6.735. Dos 145 artigos publicados em periódicos que fazem parte das referências, 35 apresentam mais de 100 citações e quatro artigos, mais de 1.000 citações. Verificou-se que os artigos que possuem mais de 1.000 citações são aqueles não diretamente relacionados ao tema de pesquisa, mas sim, artigos que tratam de conceitos mais clássicos e abrangentes, que são utilizados como suporte ao tema estudado.

Analisando conjuntamente a relevância científica dos artigos, medida pela quantidade de citações de cada artigo, e a incidência de artigos do mesmo autor presentes nas referências no portfólio bibliográfico, obtém-se a matriz de artigos de maior destaque do portfólio bibliográfico (Figura 4). Verifica-se a existência de três artigos de destaque: de G. Miller e F. Figge et al., que se destacam pela quantidade de citações acima da faixa padrão, em que se concentra a maior parte dos artigos do portfólio bibliográfico, e o artigo de V. Veleva et al., onde o fator de destaque é o autor, que possui a maior quantidade de artigos nas referências do portfólio bibliográfico. Não foram evidenciados artigos que se destacassem em ambos os critérios.

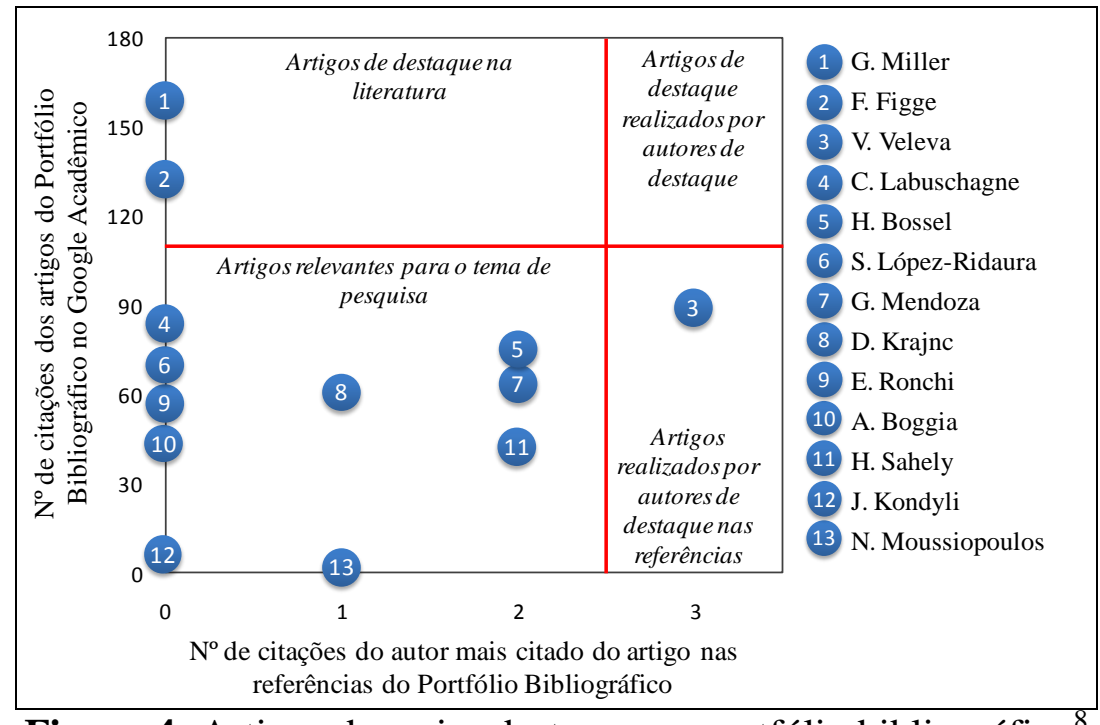

Figura 4: Artigos de maior destaque no portfólio bibliográfico ${ }^{8}$

Fonte: Resultados da pesquisa.

\footnotetext{
${ }^{8} \mathrm{O}$ ponto de corte para os dois eixos da matriz foi arbitrado pelos autores. A metodologia proposta por Ensslin, L. et al. (2010a) sugere que o ponto de corte seja estabelecido, de forma a alocar cerca de $20 \%$ dos artigos na faixa de destaque, em cada um dos eixos.
} 
A análise das palavras-chave do portfólio bibliográfico indica a ocorrência de 53 palavraschave diferentes, sendo grande parte delas combinações de palavras que contêm as palavras-chave definidas originalmente para a pesquisa. Uma palavra-chave se destaca entre as demais, estando presente em seis artigos: "sustainable development"; outras sete palavras-chave se destacam por estarem presentes em dois artigos cada (veja Figura 5).

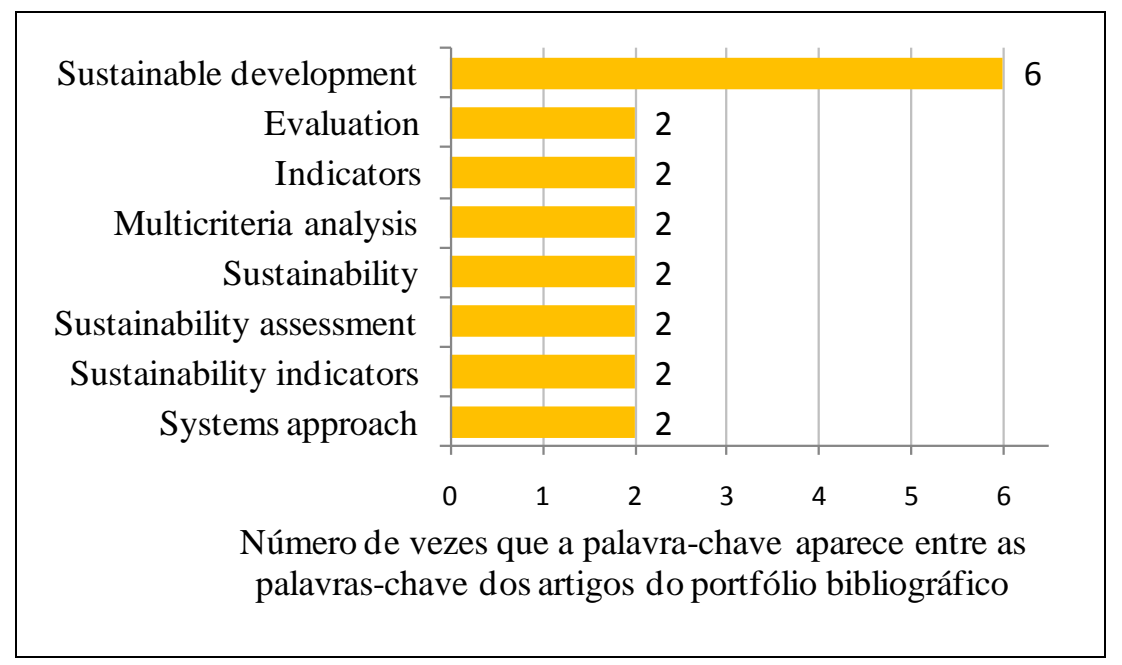

Figura 5: Palavras-chave dos artigos do portfólio bibliográfico.

Fonte: Resultados da pesquisa

\section{CONSIDERAÇÕES FINAIS}

A produção científica tem apresentado grande crescimento na última década e aponta sinais de continuar se elevando nos próximos anos. O pesquisador, ao mesmo tempo que possui maior riqueza informativa à sua disposição, se vê impossibilitado de aproveitar toda a informação disponível, sendo necessário optar, seletivamente, pelo conteúdo a ser considerado em sua pesquisa. A dificuldade de estabelecer critérios de seleção e de seguir um processo rigoroso na busca por informação relevante salientam a importância de se fazer uso de uma metodologia objetiva na seleção de referencial bibliográfico para uma pesquisa científica.

Este trabalho teve por objetivo apresentar uma metodologia estruturada para a construção de referencial teórico sobre o tema de pesquisa. Para suportar este objetivo, os seguintes objetivos específicos foram estabelecidos: 1) selecionar as palavras-chave adequadas para a pesquisa; 2) selecionar as bases de dados relativas ao tema de pesquisa; 3 ) proceder a busca de artigos alinhados com o tema de pesquisa; 4) identificar os artigos relevantes da amostra selecionada; e 5) analisar os resultados obtidos com relação a relevância científica, saliência de autores e periódicos de destaque.

O processo de seleção de portfólio bibliográfico ProKnow-C, desenvolvido pelo LabMCDA, se mostra como uma ferramenta efetiva na seleção de informação, conduzindo o pesquisador a seguir uma metodologia clara, objetiva e com rigor científico. $O$ processo busca, entre seus objetivos, gerar informação relevante minimizando a seleção de conteúdos, de forma aleatória ou parcial. No entanto, é importante considerar que o pesquisador é parte fundamental deste processo, podendo, em algumas etapas da metodologia, influenciar nos resultados obtidos por meio de seus julgamentos. Tal característica, todavia, não torna menos válida a aplicação da metodologia, uma vez que esta segue etapas determinadas e as decisões tomadas são justificadas pelo pesquisador.

O processo subsequente de análise bibliométrica se mostra como uma ferramenta de grande validade no entendimento e análise do contexto informacional da área de pesquisa, possibilitando visualizar, de forma clara, as características de produção científica acerca do tema. 
A metodologia aplicada permitiu identificar que a produção científica na área de avaliação de desempenho em desenvolvimento sustentável se constitui em um campo que tem apresentado franco crescimento na última década, sendo bastante restrita a quantidade de publicações nos anos anteriores ao período de pesquisa, ou seja, antes de 2001.

Em consonância com a constatação de se tratar de uma área ainda bastante incipiente em termos de publicações científicas, evidenciou-se que os artigos do portfólio bibliográfico obtido não apresentam uma quantidade tão elevada de citações se comparados a outras áreas mais tradicionais da ciência. Em sua grande maioria, estão na faixa de 40 a 90 citações. Dois artigos se destacam com relação à relevância científica, são os de Miller (2001) e de Figge e Hahn (2004).

Não foi possível identificar autores de destaque no portfólio bibliográfico, indicando uma grande disseminação dos artigos entre autores diferentes. Por outro lado, dois periódicos se destacam com maior presença no portfólio bibliográfico: Ecological Indicators e Jornal of Cleaner Production.

É importante considerar que os resultados obtidos estão limitados à amostra de periódicos pesquisados e às palavras-chave utilizadas pelos autores e não podem ser extrapolados para todo o conjunto de publicações da área. Para futuras pesquisas sobre o tema, recomenda-se aplicar a próxima fase da metodologia ProKnow-C, desenvolvida pelo LabMCDA, que remete à análise sistêmica do conteúdo deste portfólio bibliográfico.

\section{REFERÊNCIAS}

Boggia, A., \& Cortina, C. (2010). Measuring sustainable development using a multi-criteria model: A case study. Journal of Environmental Management, 91(11), 2301-2306.

Bortoluzzi, S. C., \& Ensslin, L. (2010). Congruências e divergências na avaliação de desempenho organizacional: análise crítica de pesquisas publicadas em periódicos nacionais e internacionais no período de 2000 a 2008. Simpósio de Administração da Produção, Logística e Operações Internacionais, 14, São Paulo, SP, Brasil.

Bossel, H. (2001). Assessing viability and sustainability: a systems-based approach for deriving comprehensive indicator sets. Conservation Ecology, 5(2): 12.

Cebds. (2009). Guia de comunicação e sustentabilidade. realização câmara temática de comunicação e educação do CEBDS. Recuperado em 16 maio, 2011, de http://www.cebds.org.br/cebds/MANUAL_DE_SUSTENTABILIDADE.pdf

Capes (2011a). Estatísticas de uso. Recuperado em 16 maio, 2011, de http://www.periodicos.capes.gov.br/index.php?option=com_pestatistics\&mn=69\&smn=77.

Capes (2011b). Quem pode usar o portal? Recuperado em 16 maio, 2011, de http://periodicos.capes.gov.br/portugues/paginaInicial/faq.htm

Delai, I., \& Takahashi, S. (2008). Uma proposta de modelo de referência para mensuração da sustentabilidade corporativa. RGSA - Revista de Gestão Social e Ambiental, 2(1), 19-40.

Echer, I. C. (2001). A revisão de literatura na construção do trabalho científico. Revista gaúcha de enfermagem, 22(2), 5-20.

Elkington, J. (1999). Cannibals with forks: the triple bottom line of $21^{\text {st }}$ century business. Capstone Publishing Limited. London: John Wiley \& Sons.

Revista de Gestão Social e Ambiental - RGSA, São Paulo, v. 5, n. 2, p. 47-62, mai./ago. 2011. 
Ensslin, L., Ensslin, S. R., Lacerda, R. T. O., \& Tasca, J. E. (2010a). Processo de análise bibliométrica. Processo técnico com patente de registro pendente junto ao INPI. Brasil.

patente de registro pendente junto ao INPI. Brasil.

(2010b). Processo de análise sistêmica. Processo técnico com .. (2010c). Processo de Seleção de Portfólio Bibliográfico.

Processo técnico com patente de registro pendente junto ao INPI. Brasil.

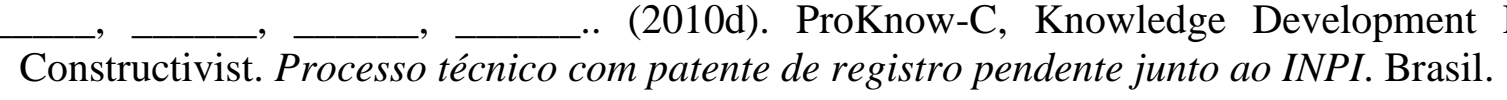

Figge, F., \& Hahn, T. (2004). Sustainable value added: measuring corporate contributions to sustainability beyond eco-efficiency. Ecological Economics, 48(2), 173-187.

Kondyli, J. (2010). Measurement and evaluation of sustainable development: A composite indicator for the islands of the North Aegean region, Greece. Environmental Impact Assessment Review, $30,347-356$.

Krajnc, D., \& Glavič, P. (2005). A model for integrated assessment of sustainable development. Resources Conservation and Recycling, 43(2), 189-208.

Labuschagne, C., Brent, A. C., \& Erck, R. P. G. van. (2005). Assessing the sustainability performances of industries. Journal of Cleaner Production, 13(4), 373-385.

Lacerda, R. T. O., Ensslin, L., \& Ensslin, S. R. (2011a). Revisão sistêmica da literatura sobre estratégia e avaliação de desempenho. Florianópolis/SC, artigo técnico do LabMCDA, Departamento de Engenharia de Produção e Sistemas, UFSC.

(2011b). Uma análise bibliométrica da literatura sobre estratégia e avaliação de desempenho. Florianópolis/SC, artigo técnico do LabMCDA, Departamento de Engenharia de Produção e Sistemas, UFSC.

Lima, T. C. S., \& Mioto, R. C. T. (2007). Procedimentos metodológicos na construção do conhecimento científico: a pesquisa bibliográfica. Katálysis, 10 (esp.), 37-45. Recuperado em 26 maio, 2011, de http://www.scielo.br/scielo.php?script=sci_arttext\&pid=S1414$49802007000300004 \& \operatorname{lng}=\mathrm{en} \& \mathrm{nrm}=$ iso.

López-Ridaura, S., Masera, O., \& Astier, M. (2002). Evaluating the sustainability of complex socioenvironmental systems. The MESMIS framework. Ecological Indicators, 2, 135-148.

Macedo, N. D. de. (1994). Iniciação à pesquisa bibliográfica: guia do estudante para fundamentação do trabalho de pesquisa (2a ed.). São Paulo: Loyola.

Mendoza, G. A., \& Prabhu, R. (2003). Qualitative multi-criteria approaches to assessing indicators of sustainable forest resource management. Forest Ecology and Management, 174(13), 329-343.

Miller, G. (2001). The development of indicators for sustainable tourism: results of a Delphi survey of tourism researchers. Tourism Management, 22(4), 351-362. 
Moussiopoulos, N., Achillas, C., Vlachokostas, C., Spyridi, D., \& Nikolaou, K. (2010). Environmental, social and economic information management for the evaluation of sustainability in urban areas: A system of indicators for Thessaloniki, Greece. Cities, 27(5), 377-384.

Penteado, R. (2005). Análise e mineração de textos e dados. Em Duarte, J., \& Barros, A. T. de. (Orgs.). Pesquisa em comunicação: métodos e técnicas. São Paulo: Atlas.

Ronchi, E., Federico, A., \& Musmeci, F. (2002). A system oriented integrated indicator for sustainable development in Italy. Ecological Indicators, 2, 197-210.

Rosa, F. S., Ensslin, S. R., \& Ensslin, L. (2009). Evidenciação ambiental: processo estruturado de revisão de literatura sobre avaliação de desempenho da evidenciação ambiental. Sociedade, Contabilidade e Gestão, 4, 4-8.

Sahely, H. R., Kennedy, C. A., \& Adams, B. J. (2005). Developing sustainability criteria for urban infrastructure systems. Canadian Journal of Civil Engineering, 32, 72-85.

Tasca, J. E., Ensslin, L., Ensslin, S. R., \& Alves, M. B. M. (2010). An approach for selecting a theoretical framework for the evaluation of training programs. Journal of European Industrial Training, 34(7); 631-655.

Trentini, M., \& Paim, L. (1999). Pesquisa em enfermagem: uma modalidade convergenteassistencial. Florianópolis: Editora da UFSC.

Valmorbida, S. M. I., Ensslin, L., \& Ensslin, S. R. (2011). Gestão pública com foco em resultados: evidenciação do estado da arte para as delimitações postas pelo pesquisador. Florianópolis/SC, artigo técnico do LabMCDA, Departamento de Engenharia de Produção e Sistemas, UFSC.

Vasconcelos, A. C. F. de, Andrade, E. O., \& Cândido, G. A. (2009). Indicadores de sustentabilidade e suas influências no desenvolvimento local: uma aplicação em região produtora de mamona no estado da Bahia. RGSA - Revista de Gestão Social e Ambiental, 2(1), 19-40.

Veleva, V., \& Ellenbecker, M. (2001). Indicators of sustainable production: framework and methodology. Journal of Cleaner Production, 9(31), 519-549.

Wced (1987). Our common future. Oxford: Oxford University Press.

Data do recebimento do artigo: 28/04/2011

Data do aceite de publicação: 18/08/2011

Revista de Gestão Social e Ambiental - RGSA, São Paulo, v. 5, n. 2, p. 47-62, mai./ago. 2011. 